\title{
Transmission spectra changes produced by decreasing compactness of opal-like structures
}

A. Andueza, R. Echeverría, P. Morales, and J. Sevilla

Citation: Journal of Applied Physics 105, 024910 (2009); doi: 10.1063/1.3068475

View online: https://doi.org/10.1063/1.3068475

View Table of Contents: http://aip.scitation.org/toc/jap/105/2

Published by the American Institute of Physics

\section{Articles you may be interested in}

Electromagnetic resonant modes of dielectric sphere bilayers

Journal of Applied Physics 117, 204903 (2015); 10.1063/1.4921826

Geometry influence on the transmission spectra of dielectric single layers of spheres with different compactness Journal of Applied Physics 107, 124902 (2010); 10.1063/1.3434529

Erratum: "Transmission spectra changes produced by decreasing compactness of opal like structures" [J. Appl. Phys. 105, 024910 (2009)]

Journal of Applied Physics 109, 019902 (2011); 10.1063/1.3524566

Evolution of the electromagnetic modes of a single layer of dielectric spheres with compactness Journal of Applied Physics 104, 043103 (2008); 10.1063/1.2969659

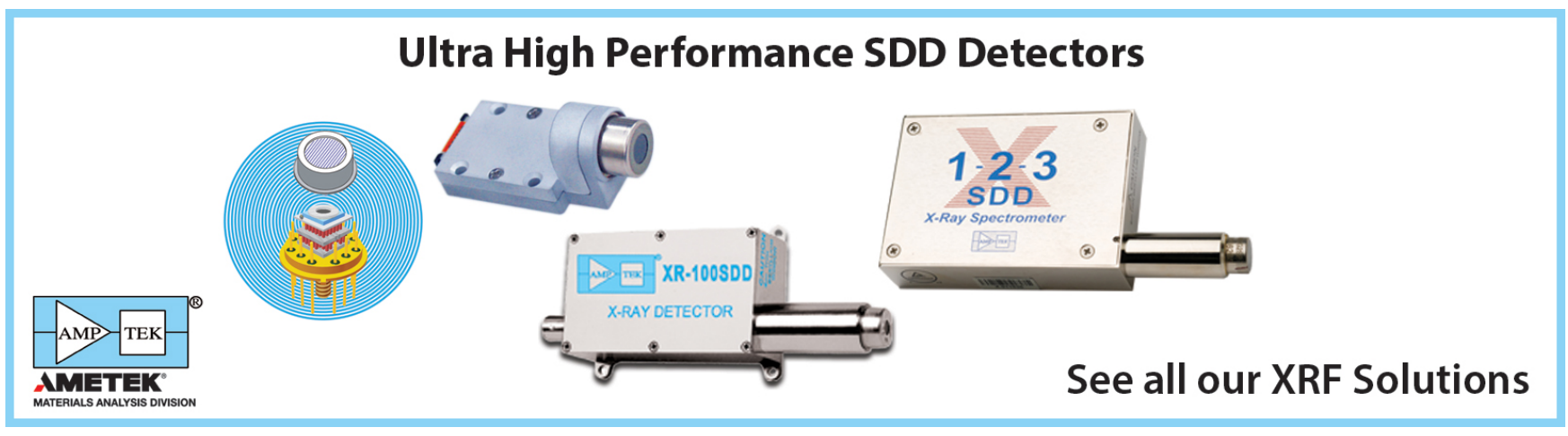




\title{
Transmission spectra changes produced by decreasing compactness of opal-like structures
}

\author{
A. Andueza, ${ }^{\text {a) }}$ R. Echeverría, P. Morales, and J. Sevilla ${ }^{\text {b) }}$ \\ Departamento Ingeniería Eléctrica y Electrónica, Universidad Pública de Navarra, Campus de Arrosadia, \\ 31006 Pamplona, Spain
}

(Received 8 October 2008; accepted 5 December 2008; published online 28 January 2009)

\begin{abstract}
Artificial opal-like structures based on spheres and colloidal particles have been fabricated in a controlled way, presenting optical band-gap properties in the optical frequency range. Nonclose packed artificial opals have also been fabricated and studied recently. In order to gain a better understanding of these phenomena, we have studied macroscopic models of nonclose packed fcc lattices using glass spheres $(\varepsilon=7)$ of $8 \mathrm{~mm}$ diameter, and measuring in the microwave region (from 10 to $30 \mathrm{GHz}$ ). The results have shown a Bragg resonance tunable with filling factor of the opal, and a strong rejected band similar, also present in close packed samples, much less affected by compactness. The relation of this high order band with spheres single layer behavior is also discussed. (C) 2009 American Institute of Physics. [DOI: 10.1063/1.3068475]
\end{abstract}

\section{INTRODUCCTION}

Three dimensional photonic crystals are very interesting materials that offer extraordinary properties and promise applications in optical and microwaves devices. ${ }^{1-3}$ This interest is based on the property of forbidding the propagation of photons of certain energies in all spatial directions produced by a periodic structuration of dielectric constant. Is the analogy with crystalline materials, forbidding certain electronic energies due to the periodicity of atomic potential, what gives rise to the name of photonic crystal or photonic band gap.

In the past 20 years, several methods to fabricate threedimensional photonic crystals have been reported, ${ }^{4-7}$ all aiming to achieve materials presenting complete band gaps. Artificial opal-like structures based colloidal spheres have been fabricated in a controlled way, ${ }^{5}$ presenting photonic bandgap properties in the optical frequency range. Most of the works published on fabrication of these structures are focused on close packed symmetries, controlling the connectivity, and topology of the dielectric medium. ${ }^{5-7}$

New methods of fabrication have recently led to experimental demonstration of nonclose packed opals. ${ }^{8-15}$ Chemical methods to achieve a nonclose packed opal, presenting a full gap have been reported, ${ }^{10}$ including a theoretical study of nonclose packed inverse opals. ${ }^{14,15}$

The aim of the work here presented to gain a better understanding of the electromagnetic behavior of non compact opals by comparing their transmission spectra with compact opal one. The samples were constructed by piling layers of silica spheres (of $8 \mathrm{~mm}$ diameter). In the noncompact case, the fist layer is firmly placed maintaining a certain separation among the spheres, while the following will keep their places by gravity. Therefore the resulting material will be composed of noncompact layers stacked closer than in the

\footnotetext{
a)Electronic mail: angel.andueza@unavarra.es.

${ }^{b)}$ Electronic mail: joaquin.sevilla@unavarra.es.
}

compact case. Normal incidence transmission spectra in the microwave region (ranging from 10 to $30 \mathrm{GHz}$ ) were measured.

In all spectra, two main features are present, a Bragg resonance, tunable with the topology of the opal, and a higher energy rejected band ${ }^{17-21}$ which remains much less affected by the compactness. We present the spectra as the layers were built up in our opals in order to clarify the origin of the high energy rejected band and how can it be related to the physical behavior of two dimensional arrays of spheres. ${ }^{16}$

\section{EXPERIMENTAL SETUP}

Spherical balls of Borosilicate (purchased from Afora S.A.) with a diameter $\Phi=8 \mathrm{~mm}$, were used for building opals like samples. They have a fairly high dielectric constant $\varepsilon=7(n=2.64)$ in the microwave frequency region investigated. A sketch of the nonclose packed sample is showing in Fig. 1. The spheres of the first layer are firmly kept at their designed positions by holes drilled in a wooden pattern [Fig. 1(b)]. Subsequent layers were directly placed over the previous and held at their places by gravity. This building process assures the spacing among the spheres present in the fist layer is maintained in all the following ones [see Fig. 1 (a)] but the spacing among layers is less than in the compact case.

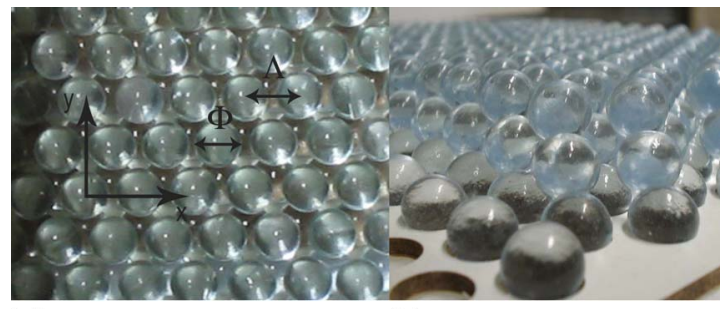

(a)

(b)

FIG. 1. (Color online) (a) Image of fabricated opal where the (111) facet can be seen. Note the small space between the spheres. (b) Image of the sample building process. 


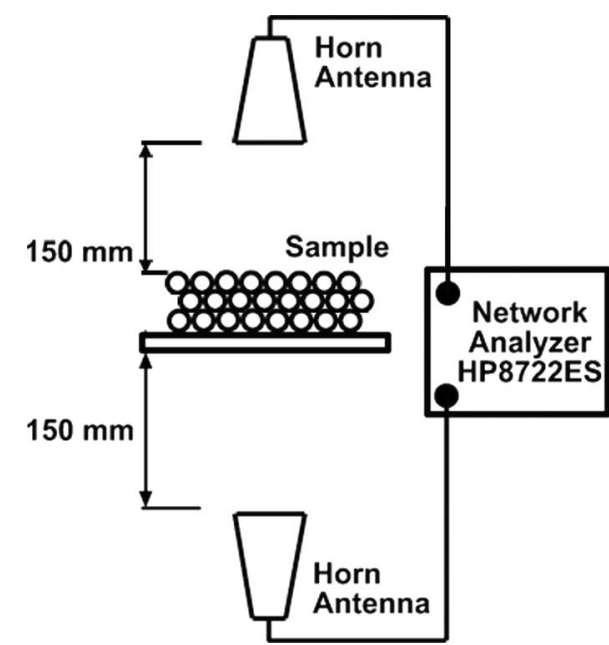

FIG. 2. Outline of the experimental setup. Radiation emitted and detected in the 111 direction of the sample.

The parameter notation is shown in Fig. 1(a): we will use $\Lambda$ to refer the lattice period, which stands for the separation between two consecutive spheres centers in the $x-y$ plane, $\Phi$ for the spheres diameter and $z$ as the piling layers direction. Two opal-like structures were built up, one close packed opal (with $\Lambda$ equal to $\Phi$ ), and a nonclose packed one with a lattice period value $\Lambda=9 \mathrm{~mm}, 1 \mathrm{~mm}$ larger than the sphere diameter.

Measurements were accomplished with a network analyzer (Agilent HP 8722ES) which provides the phase and module transmission S-parameters, spliced to rectangular horn antennas (Narda model 639). These antennas were aligned in the $z$ axis and separated $400 \mathrm{~mm}$ from each other (see Fig. 2). In the middle of this space the opal samples were placed perpendicular to the antenna axis in the $\langle 111\rangle$ direction. The antennas show polarization sensitive coupling characteristics. In all cases measurements were taken in different polarization configuration showing no appreciable differences.

Measurements were performed differentially, taking in first place the transmittance of the system without sample in the middle as reference $T_{\text {ref }}(\omega)$ and immediately after the same measurement with the spheres arrangement placed between the antennas $T_{\text {sam }}(\omega)$. Therefore the transmittance of the sphere arrangement alone $T(\omega)$ is given by $T(\omega)$ $=T_{\text {sam }}(\omega) / T_{\text {ref }}(\omega)$.

\section{RESULTS AND DISCUSSION}

The transmittance spectra for ten layers opals are shown in Fig. 3 for the $\langle 111\rangle$ direction. In order to allow clear comparison of the results with different compactness, data are presented normalized. A dimensionless frequency value is calculated from the measured wavelength $\omega$ as $\omega \Lambda / 2 \pi c$ or $\Lambda / \lambda$, where $\Lambda$ is the lattice period, $\lambda$ is the corresponding wavelength, and $c$ stands for light velocity in vacuum.

Both spectra shown in Fig. 3 present the same features but placed at different frequency values. First we can see a relatively small peak, around $\Lambda / \lambda=0,29$ in the compact case and 0,38 in the noncompact one. Besides, a sharper and

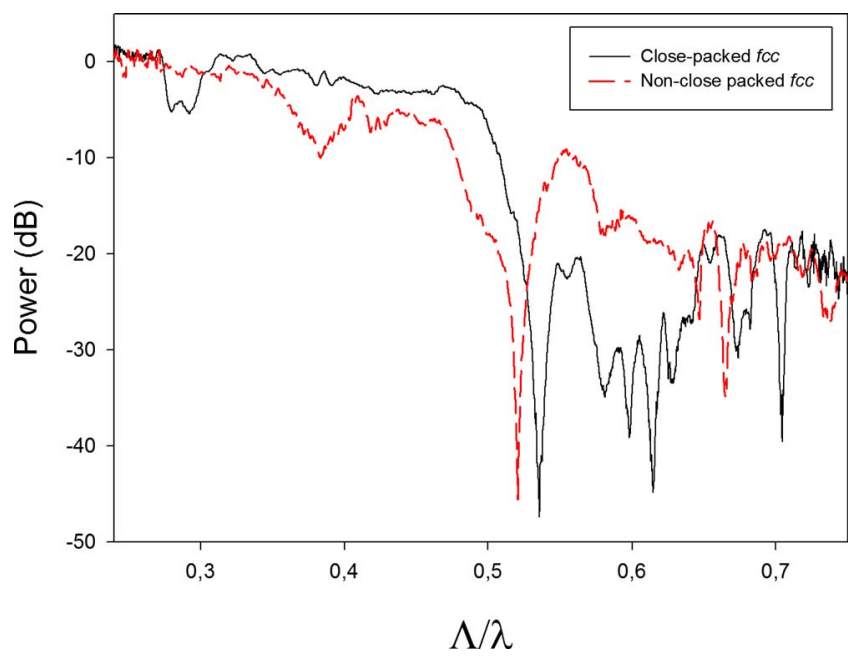

FIG. 3. (Color online) Transmission spectra of close packed (solid line) and nonclosed packed (dashed line) fcc opal-like structures of $8 \mathrm{~mm}$ glass spheres. The frequency is normalized by $\omega \Lambda / 2 \pi c$.

deeper peak can be seen (placed at $\Lambda / \lambda=0.52$ and 0.54 in each spectra). Following this strong peak a complex pattern of consecutive and non regular dips is observed. It is interesting to note that these dips are found in both cases for values of the normalized frequency relatively close, with a slightly shift to lower frequencies in the noncompact case, the opposite direction than observed in first reported peak.

As a first approach to explain the observed peaks in the spectra, we can propose a simple model for the Bragg scattering. ${ }^{12}$ This model considers that the incident radiation is partly reflected at the planes passing through the spheres centers (separated form each other a distance $d_{111}$ ) and that the material presents a refractive index average of the air of the interstices and the glass of the spheres. With these assumptions, in the case of normal incidence, the first-order Bragg diffraction is given by:

$$
\lambda=2 d_{111}\left[n_{s}^{2} \mathrm{ff}+n_{a}^{2}(1-\mathrm{ff})\right]^{1 / 2},
$$

where $\lambda$ is the wavelength, $d_{111}$ is the distance between spheres planes in $\langle 111\rangle$ direction, $n_{s}$ and $n_{a}$ are the refractive indices of the spheres and the surrounding medium respectively and ff is the filling factor of the opal.

The distance $d_{111}$ between two consecutive sphere layers can be derived from simple geometrical considerations. Following Fig. 4 we can see how the centers of three adjacent

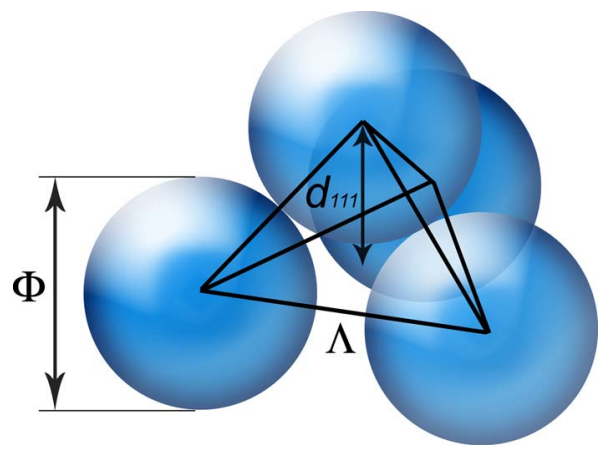

FIG. 4. (Color online) Sketch of four spheres in the noncompact opal useful to calculate the Bragg reflection frequency. 
spheres in the lower plane form a equilateral triangle whose side equals $\Lambda$. The center of a sphere in the upper plane completes a tetrahedron with the abovementioned triangle. As this sphere touches the other three, the length of the three new sides equals $\Phi$. Solving the height of this figure, it is found

$$
d_{111}=\left[\Phi^{2}-\Lambda^{2} / 3\right]^{1 / 2}
$$

In the case of the close packed opal, $\Lambda=\Phi$, therefore $d_{111}$ results

$$
d_{111}=\left[2 \Phi^{2} / 3\right]^{1 / 2}
$$

In the case of the noncompact one the lattice parameter is larger than the sphere diameter. Therefore the distance between planes $d_{111}$ is different, it decreases as the lattice parameter increases.

The ff is also different in the close packed and the noncompact cases. It can be derived from simple geometrical calculations following Fig. 4. For the noncompact case he result is

$$
\mathrm{ff}=(\pi / 3 \sqrt{2})[\Phi / \Lambda]^{3} n
$$

The value obtained for the samples used in our measurement are $\mathrm{ff}_{\mathrm{nc}}=0.52$ for the non compact opal, significantly smaller than the close packed value $\mathrm{ff}_{c}=0.74$.

Putting together the values of $\mathrm{ff}_{\mathrm{nc}}$ [Eq. (4)], and $d_{111}$ [Eq. (2)] in the Bragg law [Eq. (1)] and applying the above mentioned normalization, we find a Bragg reflection value given by

$$
\frac{\Lambda}{\lambda}=\frac{\Lambda}{2 \sqrt{\left(\Phi^{2}-\frac{\Lambda^{2}}{3}\right)\left(n_{s}^{2} \mathrm{ff}_{\mathrm{nc}}+n_{a}^{2}\left(1-\mathrm{ff}_{\mathrm{nc}}\right)\right)}} .
$$

Equation (5) can be solved in the particular case of the close packed opal, $\Phi=\Lambda$, finding a Bragg reflection given by

$$
\frac{\Lambda}{\lambda}=\frac{\Phi}{2 \sqrt{\left(\frac{2 \Phi^{2}}{3}\right)\left(n_{s}^{2} \mathrm{ff}_{c}+n_{a}^{2}\left(1-\mathrm{ff}_{c}\right)\right)}} .
$$

Following Eqs. (5) and (6), Bragg diffraction peaks are expected at $\Lambda / \lambda \sim 0.365$ and $\Lambda / \lambda \sim 0.263$ in nonclose packed and close packed opals, respectively. Therefore, we can attribute the lower frequency peak (experimentally observed around 0.38 and 0.28 ) to the Bragg reflection generated by spheres layers. Calculated and measured values only differ in a $4 \%$ and $6 \%$, respectively, which can be due both to model limitations and to finite size and imperfections of the opal samples.

As the spheres become separated in one layer, the following one fits at a lower positions. So the Bragg reflecting planes become closer and the reflected wavelength decreases. This leads to an increase in the corresponding frequency, as experimentally observed. However, the high order rejected band cannot be explained as Bragg scattering. It does not correspond to higher orders of this phenomenon, and the observed shift in frequency takes place in the opposite direction.

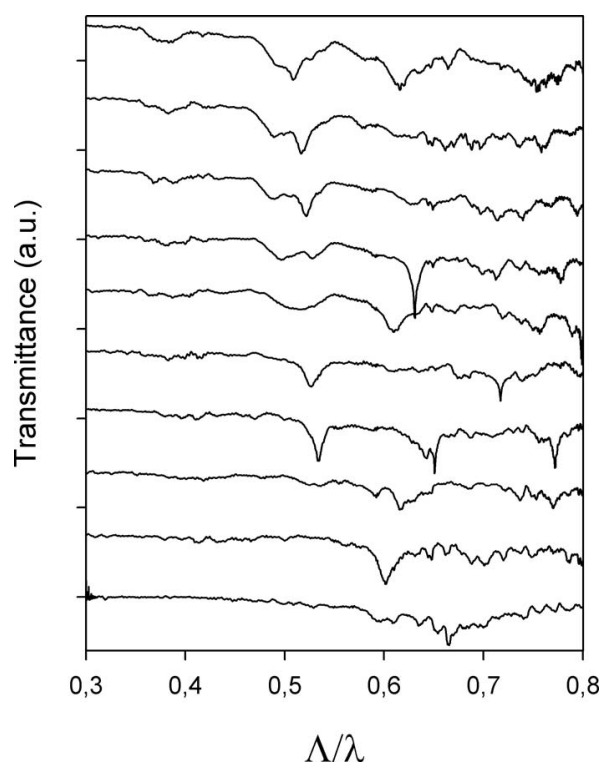

FIG. 5. Transmission spectra of different numbers of layers from nonclosed packed fcc opal-like structure of $8 \mathrm{~mm}$ soda-lime spheres. Layer 1 (the lowest) through 10 (upper line).

In Fig. 5 the transmission spectra fcc nonclose packed opal of $8 \mathrm{~mm}$ diameter spheres are presented for a number of stacked layers ranging from 1 to 10 . It can be seen how the 0.38 peak first described start to be appreciable for seven or eight layers and needs ten to be fully developed. This fact is consistent with the Bragg origin previously attributed to this peak. Several of these planes are needed to have the phenomenon observable, and it increases with the number of layers (Bragg reflectors) as is expected.

In the frequency region of the high rejected band, even for only one layer, some peaks can be observed in the spectrum. ${ }^{16}$ However, as the number of layers increases, these peaks shift to lower frequencies and some others appear at different values. Spheres single layers show a transmission peak pattern crated by collective resonances that take place for particular values of the incident radiation frequency. These resonances are produced by non trivial combinations of individual sphere resonances (Mie modes) and collective Bragg resonances. ${ }^{22-26}$ In our previous work we have studied the evolution of the resonance pattern with the layer compactness ${ }^{16}$ and the results show a general shift to lower frequencies as compactness decreases. For compactness ratios of $\Phi / \Lambda$ down to 0.75 the frequency shift is very weak, increasing for lower values. As this band is developed over the resonances of a single layer of spheres, this result is consistent with the resonance frequency decrease produced in the single layer by decreasing compactness.

\section{CONCLUSIONS}

We have presented a study of the effect of compactness in the electromagnetic transmission of nonclose packed fcc opal-like arrangements of glass spheres. Experiments were performed in the microwave domain (from 10 to $30 \mathrm{GHz}$ ) with glass spheres of $8 \mathrm{~mm}$ diameter of high dielectric permittivity $(\varepsilon=7)$. 
The spectra of the compact and the noncompact samples present the same features but shifted in their frequency values. A first peak, which shifts to higher frequencies as compactness decreases, is attributed to Bragg reflection originated by spheres planes. A high order rejected band shifts slightly in the opposite direction than the Bragg peak. This behavior is consistent with previously reported data on the behavior of spheres single layers, considering that the band is generated by adding complexity to the single layer behavior introducing further interactions between spheres in different planes.

\section{ACKNOWLEDGMENTS}

The authors wish to acknowledge F. Meseguer, R. Fenollosa, and M. Rubi for their support and valuable discussions.

${ }^{1}$ E. Yablonovitch, Phys. Rev. Lett. 58, 2059 (1987).

${ }^{2}$ S. John, Phys. Rev. Lett. 58, 2486 (1987).

${ }^{3}$ J. D. Joannopoulos, Photonic Crystals: Molding the Flow of Light (Princeton University Press, New Jersey, 1995).

${ }^{4}$ P. V. Braun and P. Wiltzius, Nature (London) 402, 603 (1999).

${ }^{5}$ J. E. G. J. Wijnhoven and W. L. Vos, Science 281, 802 (1998).

${ }^{6}$ A. Blanco, E. Chomski, S. Grabtchak, M. Ibisate, S. John, S. W. Leonard, C. Lopez, F. Meseguer, H. Miguez, J. P. Mondla, G. A. Ozin, O. Toader, and H. M. Van Driel, Nature (London) 405, 6785 (2000).

${ }^{7}$ H. Míguez, F. Meseguer, C. López, A. Mifsud, J. S. Moya, and L. Vázquez, Langmuir 13(23), 6009 (1997).
${ }^{8}$ F. Meseguer, Colloids Surf. 270-271, 1 (2005).

${ }^{9}$ R. Fenollosa and F. Meseguer, Adv. Mater. (Weinheim, Ger.) 15, 1282 (2003).

${ }^{10}$ F. Meseguer and R. Fenollosa, J. Mater. Chem. 15, 4577 (2005).

${ }^{11}$ Z. Ren, X. Zhang, J. Zhang, X. Li, X. Pan, X. Fei, Z. Cui, and B. Yang, J. Mater. Chem. 18, 3536 (2008).

${ }^{12}$ F. García-Santamaría, and P. V. Braun, Appl. Phys. Lett. 90, 241905 (2007).

${ }^{13}$ J. Li, S. Zhang, H. Chen, Z. Gu, and Z. Lu, Colloids Surf., A 299, 54 (2007).

${ }^{14}$ D. P. Gaillot and C. J. Summers, J. Appl. Phys. 100, 113118 (2006).

${ }^{15}$ J. S. King, D. P. Galliot, E. Graugnard, and C. J. Summers, Adv. Mater. (Weinheim, Ger.) 18, 8 (2006)

${ }^{16}$ A. Andueza, R. Echeverría, and J. Sevilla, J. Appl. Phys. 104, 043103 (2008).

${ }^{17}$ A. Andueza and J. Sevilla, Opt. Quantum Electron. 39, 311 (2007).

${ }^{18}$ Y. A. Vlasov and D. J. Norris, Evolution of the Higher Order Band Structure in FCC Photonic Crystals, Proceedings of CLEO/QELS 2001, Baltimore, MD, May 2001, p. 588.

${ }^{19}$ D. J. Norris and Yu. A. Vlasov, Photonic Crystals and Light Localization in the 21st Century, edited by C. M. Soukoulis (Kluwer, Dordrecht, 2001), p. 229.

${ }^{20}$ H. Míguez, V. Kitaev, and G. A. Ozin, Appl. Phys. Lett. 84, 8 (2004).

${ }^{21}$ G. Von Freymann, S. John, S. Wong, V. Kitaev, and G. A. Ozin, Appl. Phys. Lett. 86, 053108 (2005).

${ }^{22}$ K. Ohtaka and M. Inoue, Phys. Rev. B 25, 677 (1982).

${ }^{23}$ K. Ohtaka, Phys. Rev. B 19, 5057 (1979).

${ }^{24}$ H. T. Miyazaki, H. Miyazaki, K. Ohtaka, and T. Sato, J. Appl. Phys. 87, 7152 (2000).

${ }^{25}$ Y. Kurokawa, Y. Jimba, and H. Miyazaki, Phys. Rev. B 70, 155107 (2004).

${ }^{26}$ T. Kondo, M. Hangyo, S. Yamaguchi, S. Yano, Y. Segawa, and K. Ohtaka, Phys. Rev. B 66, 033111 (2002). 